\title{
Suscetibilidade de Lymnaea columella Say, 1817 à infecção por Fasciola hepatica L., 1758
}

\section{The susceptibility of Lymnaea columella Say, 1817 to the infection with Fasciola hepatica L., 1758}

\author{
Delir Corrêa Gomes, ${ }^{*}$ Nicolau Maués da Serra-Freire ${ }^{\star \star}$
}

\begin{abstract}
Resumo
São apresentadas as espécies Lymnaea columella Say, 1817 da família Lymnaeidae Rafinesque e Stenophysa marmorata (Guilding, 1828) da família Physidae Fitzinger, como as únicas espécies dos respectivos gêneros, encontradas no Estado do Rio de Janeiro. Após a identificação das espécies que ocorriam neste Estado, o estudo foi direcionado para a identificação das espécies de moluscos que possuíam real potencial como hospedeiro intermediário de Fasciola hepatica L., 1758, avaliação da suscetibilidade a miracídios de $F$. hepatica, bem como fatores que influenciavam na infecção e na ação das formas larvais do trematódeo sobre o molusco. As observações demonstraram que: L. columella foi encontrada naturalmente infectada por $F$. hepatica, tendo-se obtido êxito nas infecções experimentais, o que não ocorreu com $S$. marmorata; infecções com três miracídios/ molusco são ideais para obtenção de maior número de moluscos sobreviventes infectados e que infecções com cinco miracídios/molusco ou mais, podem produzir infecções mais intensas, porém ocasionam morte precoce; o maior número de metacercárias foi obtido nas infecções com três miracídios; L. columella não resiste a infecções com 40 ou mais miracídios; a proveniência dos ovos de $F$. hepatica com relação aos hospedeiros vertebrados (Bos taurus L. e Ovis aries L.) não influenciou nos resultados obtidos nas infecções.
\end{abstract}

Palavras-chave: suscetibilidade; F. hepatica; L. columella; S. marmorata.

\begin{abstract}
The lymnaeid Lymnaea columella Say, 1817 and the physid Stenophysa marmorata (Guilding, 1828) are the only species of these genera occurring in the State of Rio de Janeiro, Brazil. After their identification, studies were conducted in order to ascertain which of these snail species was really a potencial host to Fasciola hepatica L., 1758, their susceptibility to miracidia of $F$. hepatica, factors affecting the infection, as well as some aspects related to the action of trematode larval forms. It was demonstrated that $L$. columella was found naturally infected with $F$. hepatica and proved, experimentally, to be susceptible to infection, while $S$. marmorata was not. Three miracidia/snail is the ideal number to provide the survival of a great number of infected snails, while five or more miracidia/snail, produce intense infections with early deaths. The results obtained during the experimental infections were not affected by the origin of the eggs, either from Bos taurus L. or Ovis aries $\mathrm{L}$. The greatest number of metacercariae was obtained during infections with three miracidia/snail. $L$. columella was unable to survive when infected with 40 or more F. hepatica miracidia.
\end{abstract}

Keywords: susceptibility; F. hepatica; L. columella; S. marmorata.

\section{Introdução}

Leuckart (1882) e Thomas (1883) foram os primeiros a estudar o ciclo da Fasciola hepatica L., 1758. Em trabalhos concomitantes eles identificaram Lymnaea truncatula (Müller) como hospedeiro intermediário, descrevendo suas formas larvais.

Embora o ciclo da $F$. hepatica tenha sido o primeiro a ser descrito entre os trematódeos, para muitas regiões como 0 Brasil, faltam ainda certos conhecimentos sobre o relacionamento deste helminto com o hospedeiro intermediário, bem como os fatores que possam influenciar no relacionamento parasito/hospedeiro. De acordo com Boray (1967), a F. hepatica não foge à regra geral dos trematódeos digenéticos que são mais específicos ao hospedeiro intermediário do que ao definitivo. Segundo Shiff (1994), o parasito precisa detectar a espécie de hospedeiro apropriado, invadir e enfrentar com sucesso os mecanismos de defesa deste.

Com base na revisão bibliográfica, pode-se comprovar que no Brasil, os trabalhos sobre o relacionamento entre as formas larvais da F. hepatica e seus hospedeiros intermediári-

\footnotetext{
*Laboratório de Helmintos Parasitos de Vertebrados, Departamento de Helmintologia / IOC / Fiocruz - Av. Brasil 4365, CEP 21045-900 - Rio de Janeiro, RJ, Brasil. E-mail: dcgomes@gene.dbbm.fiocruz.br

**Laboratório de Ixodides, Departamento de Entomologia / IOC / Fiocruz. Bolsistas do CNPq, Proc. № 303124 / 89-0 e 301233 / 79-3 respectivamente.
} 
os são ainda incipientes. Quais as razões que influenciam no fato de uma espécie hospedeira ser mais susceptível, porém eliminar menor número de cercárias? Os fisídeos participam do ciclo da F. hepatica, como já foi assinalado por outros autores?

Assim, foi proposta uma análise de alguns aspectos deste relacionamento parasito-hospedeiro; avaliação sobre as espécies de caramujos, entre as encontradas em locais onde ocorre a F. hepatica no Estado do Rio de Janeiro, que possuam real potencial como hospedeiro intermediário e quais os fatores que influenciam no êxito dessa infecção. Tais proposições objetivam ampliar o conhecimento das relações parasito-hospedeiro, em qualquer fase do ciclo biológico nesta parasitose.

\section{Material e métodos}

\section{Coleta e manutenção do hospedeiro intermediário}

Durante oito meses, moluscos dos gêneros Lymnaea e Stenophysa foram procurados nas margens de riachos no Estado do Rio de Janeiro, Brasil. As coletas foram feitas nos municípios de Itaguaí e Nova Iguaçu na Zona Fisiográfica da Baixada do rio Guandu; Mendes, Vassouras, Valença, Paraibuna, Três Rios, Miguel Pereira, Rio das Flores, Barra do Piraí, Volta Redonda, Barra Mansa e Resende na Zona Fisiográfica de Resende e Friburgo, Petrópolis e Teresópolis na Zona Fisiográfica do Alto da Serra. Os exemplares de $L$. columella Say, 1817 e S. marmorata (Guilding, 1828) coletados, foram transferidos para Estação para Pesquisas Parasitológicas W. O. Neitz, do Curso de Pós-Graduação em Medicina Veterinária-Parasitologia Veterinária, Universidade Federal Rural do Rio de Janeiro, RJ, e mantidos em tanques de fibra de amianto com capacidade para mil litros de água. Os moluscos realizavam posturas em placas de isopor com $6,0 \times 4,0 \times 0,5 \mathrm{~cm}$, deixadas aleatoriamente dentro dos tanques. As placas com as posturas eram identificadas e transferidas para novos tanques previamente limpos e cheios d'água sem cloro. Os tanques que continham as colônias situavam-se do lado de fora dos laboratórios, em área coberta por árvores, protegidos por cobertura com armação de madeira com plástico na parte superior, que permitia a passagem de luz, tendo lateralmente tela de náilon para aeração, com malha de 2,0 mm. Assim foi dada origem e mantida a colônia trabalhada.

\section{Obtenção de ovos e de miracídios de F. hepatica}

Os ovos foram obtidos de posturas dos helmintos nos canais biliares dos hospedeiros vertebrados (bovino: Bos taurus L. e ovino Ovis aries L.); para isso a vesícula biliar foi amarrada ao nivel do canal biliar, seccionada e o lavado obtido foi diluído em $250 \mathrm{ml}$ de água destilada em cálice de Hoffman, permanecendo em repouso por 30 minutos para sedimentação, repetindo-se as diluições e sedimentações até que o sobrenadante permanecesse límpido e em seguida a suspensão de ovos foi passada em tamis de bronze com malha de $250 \mu \mathrm{m}$ para retirada de fragmentos de cálculos biliares e recolhidos em tamis de bronze com malha de $37 \mu \mathrm{m}$. Desse tamis, os ovos foram retirados e ressuspensos em $250 \mathrm{ml}$ de água destilada, sendo estocados em geladeira ou colocados para incubação. Os ovos provenientes dos trematódeos adultos removidos dos canais biliares ou da própria vesícula, obtidos pela dissecação do útero dos exemplares grávidos, passaram pelo mesmo procedimento.

Os ovos que se destinavam à incubação foram imersos em $100 \mathrm{ml}$ de água destilada dentro de frasco Erlenmeyer com capacidade para $250 \mathrm{ml}$, com aeração permanente, envolvido com papel alumínio e mantido à temperatura ambiente com média de $27 \pm 2^{\circ} \mathrm{C}$ e valores paramétricos entre 13 e $39^{\circ} \mathrm{C}$ para todo ano. O tempo de incubação variou de 10 a 16 dias. Após este período, o conteúdo do Erlenmeyer era distribuído em placas de Petri de $100 \times 20 \mathrm{~mm}$ e expostos à luz para eclosão dos miracídios.

\section{Infecção do hospedeiro intermediário e obtenção de metacercárias}

Foram utilizados moluscos com concha de 3 ou de $5 \mathrm{~mm}$ de comprimento nascidos nos tanques. Para as infecções, utilizaram-se placas de plástico transparente com seis orifícios cônicos de $12 \mathrm{~mm}$ de diâmetro e capacidade para $3,2 \mathrm{ml}$ de líquido; em cada compartimento era colocado um molusco e dois mililitros de água destilada. Então, cada molusco era exposto a três ou cinco miracídios recém-eclodidos, com exposição à luz pelo período de duas horas à temperatura de 27 a $29^{\circ} \mathrm{C}$; durante todo o tempo de tentativa de infecção cuidou-se para que os moluscos permanecessem sempre imersos. Após este período, verificava-se se havia ocorrido a penetração dos miracídios, e em seguida, os moluscos eram transferidos para aquários de vidro de $20 \times 15 \times 15 \mathrm{~cm}$ com dois litros de água destilada, com aeração permanente e alimentados com alface. Semanalmente era procedida a limpeza do aquário por meio de sifão, trocando-se a água. A capacidade de postura era verificada com um número de cinco moluscos colocados em aquários redondos, com $16 \mathrm{~cm}$ de diâmetro, com capacidade de $500 \mathrm{ml}$ de água destilada, dispensando-se o mesmo manejo dos outros aquários. As posturas eram feitas em placas de isopor com $6,0 \times 4,0 \times 0,5 \mathrm{~cm}$ deixadas flutuando aleatoriamente no meio líquido. Diariamente era contado o número de posturas e ovos e substituídas as placas por outras novas. Os moluscos infectados e o grupo controle foram mantidos dentro do laboratório, em temperatura ambiente que variou de 27 a $29^{\circ} \mathrm{C}$, com variação anual entre 13 e $39^{\circ} \mathrm{C}$.

Com base no desenvolvimento do projeto piloto, reconheceuse que as $L$. columella infectadas com $F$. hepatica sobreviviam no máximo 60 dias pós-infecção; assim, determinou-se que o momento ideal para avaliações da infecção era o quinquagésimo sexto dia.

A partir do qüinquagésimo sexto dia após a penetração dos miracídios nos molucos, os que apresentavam cercárias no interior da concha, identificados com auxílio de microscópio estereoscópico WILD M5, foram transferidos individualmente para placas de Petri de $100 \times 20 \mathrm{~mm}$, com fundo revestido com papel celofane incolor, umedecido com água destilada, onde eram dissecados para liberação das cercárias sob as condições de temperatura ambiente. As cercárias liberadas se encistavam sobre o papel celofane no fundo da placa, sendo contado o número de metacercárias formadas. Tentativas de obtenção de metacercárias pela liberação espontânea de cercárias, ou provocadas por choque térmico e/ ou luminoso foram realizadas. 


\section{Análise estatística}

Empregou-se estatística demonstrativa para facilitar a demonstração dos fenômenos. Recorreu-se à análise de variança ("ANOVA") e cálculos de diferença mínima significativa entre médias (DMS) pelo teste " $\mathrm{t}$ " a níveis de $95 \%$ e $99 \%$ de segurança nas afirmações, para enfatizar possíveis diferenças biológicas.

Quando o " $t$ " calculado era maior que o " $t$ " tabelado a $95 \%$ de segurança de acerto, o resultado foi considerado de diferença significativa ( $\left.{ }^{*}\right)$; quando era maior que o "t" tabelado a $99 \%$ de segurança de acerto, o resultado foi considerado de diferença altamente significativa $\left({ }^{\star \star}\right)$; quando o "t" calculado era menor que o " $\mathrm{t}$ " tabelado a $95 \%$ o resultado foi considerado de diferença não significativa (ns) (Spiegel, 1976).

\section{Resultados}

Identificação do hospedeiro intermediário - Nos oito meses de investigação com relação aos hospedeiros intermediários de $F$. hepatica nos municípios das três Zonas Fisiográficas do Estado do Rio de Janeiro, representantes das famílias Lymnaeidae Rafinesque e 1000 Physidae Fitzinger foram dissecados, sendo que Lymnaea columella Say, 1817 da primeira e Stenophysa marmorata (Guilding, 1828) da segunda, as únicas espécies encontradas.

A única espécie encontrada naturalmente infectada foi $L$. columella e somente no vale do rio Paraíba, na Zona Fisiográfica de Resende.

As tentativas de infecções experimentais com três e cinco miracídios por molusco demonstraram que $S$. marmorata não se infectou e que $46 \%$ das $L$. columella adquiriram a infecção e permitiram a multiplicação do helminto (Tabela 1).

Tabela 1 - Resultado da infecção de Stenophysa marmorata e Lymnaea columella com trêsmiracídios recém-eclodidos de Fasciola hepatica aos 56 dias pós-infecção em condições de laboratório

\begin{tabular}{lccccc}
\hline Espécie molusco & $\begin{array}{c}\text { Tamanho } \\
(\mathbf{m m})\end{array}$ & $\mathbf{N}^{\mathbf{0}}$ & $\begin{array}{c}\text { Tamanho médio } \\
\text { final }(\mathbf{m m})\end{array}$ & $\begin{array}{c}\text { Sobreviventes } \\
(\mathbf{\%})\end{array}$ & $\begin{array}{c}\text { Infectados } \\
(\mathbf{\%})\end{array}$ \\
\hline S. marmorata & $3-5$ & 50 & 9,8 & 74 & 0 \\
L. columella & $3-5$ & 50 & 9,5 & 58 & 46 \\
\hline
\end{tabular}

e bovinos. Os dados obtidos com ovos provenientes de ovinos revelou em valores corrigidos para 100 que a média de moluscos infectados foi de 17,67 com desvio-padrão de \pm 14,82 . Para os provenientes de bovinos, a média foi de 21,33 com desvio-padrão de $\pm 16,65$ moluscos. No entanto, o teste " $\mathrm{t}$ " (" $\mathrm{t}$ " calculado $=0,34 \mathrm{~ns}$ ) demonstrou que as diferenças observadas nos valores absolutos são obra do acaso, ou seja, não há diferença significativa entre as infecções com miracídios provenientes dos hospedeiros definitivos, bovinos e ovinos.

A avaliação da influência entre as infecções com três e com cinco miracídios sobre o total de metacercárias produzidas comprovou que há diferença, sendo que o maior número determina alterações mais intensas, apresentando limitações em suas fases de rédias e cercárias, resultando na menor produção de metacercárias (Figura 1).

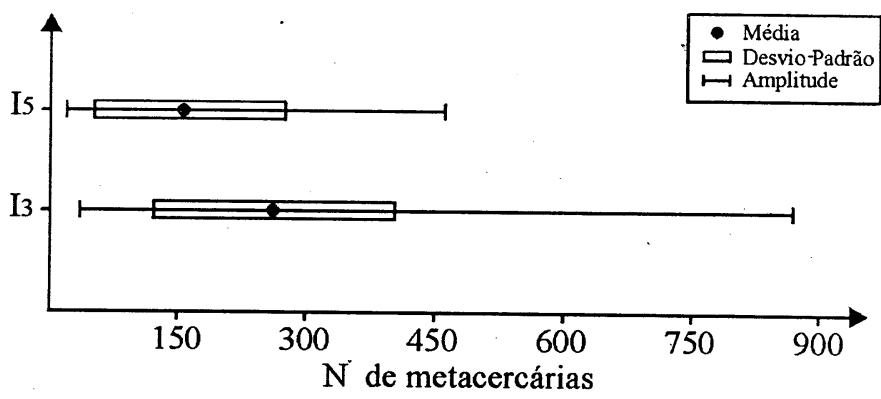

Figura 1 - Comparação da obtenção de metacercárias de $F$. hepatica aos 56 dias pós-infecção em $L$. columella com três (I3) e cinco (I5) miracídios recém-eclodidos

As infecções aos 56 dias após a penetração com três miracídios/molusco permitiram ou induziram o crescimento mais rápido dos moluscos sobre o grupo infectado com cinco, que com frequência morrem mais precocemente ( $" \mathrm{t}$ " calculado $=2,69^{\star \star}$ ). Comparados com os não infectados, a diferença para os com cinco miracídios foi significativa ("t" calculado $=1,41^{\star}$ ), mas a diferença foi altamente significativa para os com três miracídios (" $t$ " = calculado $=6,56^{\star \star}$ ), sendo a média e a moda desses muito maior (Figura 2).

Suscetibilidade de L. columella - Comprovado que somente $L$. columella se infectava com miracídios de $F$. hepatica, testou-se a suscetibilidade à infecção com três e cinco miracídios por molusco.

Corrigidos os valores obtidos para 100, calculou-se que a média com três miracídios/molusco foi de 26,17 , com desviopadrão de $\pm 13,67$ moluscos. O mesmo cálculo foi feito para cinco miracídios/molusco obtendo-se a média de 11,00, com desvio-padrão de $\pm 11,98$ moluscos. Comprovou-se pelo teste " $\mathrm{t}$ " (" $\mathrm{t}$ " calculado $=2,03^{\star \star}$ ) que as diferenças observadas entre as duas infecções eram altamente significativas, demonstrando que o maior número de miracídios/molusco promoveu infecções mais intensas com morte precoce.

Para verificar se havia alguma influência do hospedeiro vertebrado do qual provinham os ovos de $F$. hepatica, analisaramse pelo "ANOVA" e "DMS" os dados obtidos com os de ovinos

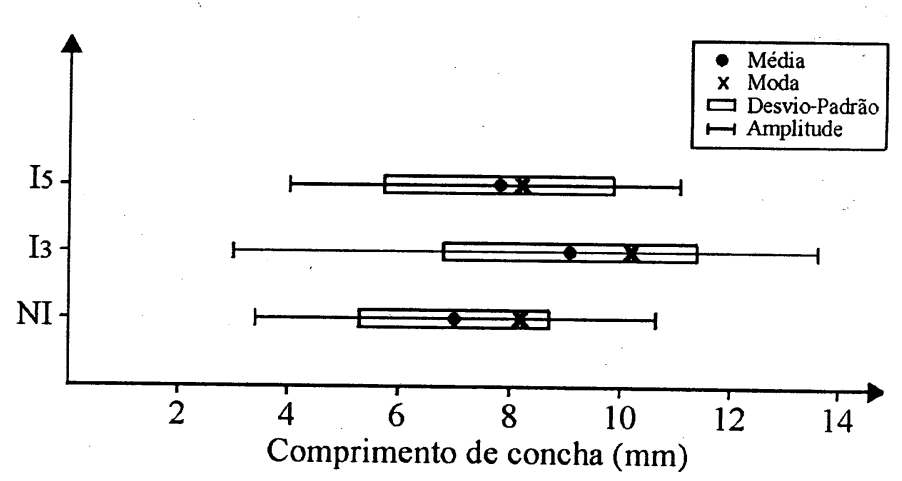

Figura 2 - Comparação do comprimento da concha de L. columella aos 56 dias entre os não infectados (NI) e infectados com três (I3) e cinco (I5) miracídios recém-eclodidos de $F$. hepatica 
As tentativas de infecção com grande número de miracídios demonstraram que os moluscos não resistem ao múltiplo desenvolvimento das formas jovens (Tabela 2).

Tabela 2 - Resultado das infecções de Lymnaea columella com 5-200 miracídios recém-eclodidos de Fasciola hepatica aos 56 dias pós-infecção em condições de laboratório

\begin{tabular}{ccccc}
\hline $\begin{array}{c}\mathbf{N}^{\mathbf{0}} \\
\text { moluscos }\end{array}$ & $\begin{array}{c}\mathbf{N}^{\mathbf{0}} \\
\text { miracídios }\end{array}$ & Sobreviventes & $\begin{array}{c}\text { Sobreviventes } \\
\text { infectados }\end{array}$ & $\begin{array}{c}\text { Infectados } \\
(\mathbf{\%})\end{array}$ \\
\hline 30 & 5 & 12 & 5 & 16,67 \\
30 & 10 & 6 & 2 & 6,67 \\
30 & 20 & 7 & 2 & 6,67 \\
30 & 30 & 5 & 1 & 3,33 \\
30 & 40 & 5 & 0 & 0 \\
30 & 50 & 3 & 0 & 0 \\
30 & 100 & 5 & 0 & 0 \\
30 & 200 & 0 & 0 & 0 \\
\hline
\end{tabular}

A comparação da produção de ovos, entre os não infectados e os infectados com cinco miracídios/molusco, demonstrou que houve uma diferença significativa para o número de ovos/ massa ovígera ( $\mathrm{t}$ " calculado $=2,88^{*}$ e para o número médio de massas ovígeras (" $t$ " calculado $=1,42^{*}$ ), havendo também diferença significativa ("t" calculado $=1,47^{*}$ ) para o número de moluscos que realizaram postura. No grupo não infectado a média de dias de postura foi significativamente (" $t$ " calculado $=1,78^{\star}$ ) maior que a média do grupo infectado.

\section{Discussão e conclusões}

Depois dos estudos de Leuckart (1882) e Thomas (1883), os pesquisadores têm-se preocupado em identificar os hospedeiros intermediários e responsáveis por parte da dispersão da $F$. hepatica nos países em que esta parasitose ocorre.

Se, por um lado, comprovaram-se os resultados de Rezende et al. (1973), demonstrando a ocorrência e a participação de L. columella no ciclo da F. hepatica no Estado do Rio de Janeiro, por outro lado não se confirmou a ocorrência de $L$. cubensis Pfeiffer, não sendo possível comprovar o envolvimento dessa espécie no ciclo desse trematódeo, como foi referido por Nuernberg et al. (1983). Contudo, esses resultados estão de acordo com Paraense (1982), que considerou L. cubensis como sinônimo de $L$. viatrix d'Orbigny e afirmou que essa espécie não ocorria no Estado do Rio de Janeiro.

O fato de nenhuma $S$. marmorata capturada apresentar-se naturalmente infectada e o resultado negativo nas infecções experimentais foi coerente com a maioria da literatura especializada, principalmente a de Amato et al. $(1985,1986)$ que estudaram durante cinco anos a epidemiologia da fasciolose hepática no Vale do Paraíba e observaram a ocorrência simultânea dessas espécies de moluscos e nunca encontraram fisídeos infectados por $F$. hepatica. A ausência de infecção ou infecção abortiva dos fisídeos são relatadas por Barthe e Rondelaud (1986). Essas observações podem ser explicadas com base na opinião de Wilson (1968) sobre 0 poder atrativo em potencial do muco de $L$. truncatula para miracídios de F. hepatica. Tais atrativos seriam certos ácidos graxos que estimulariam pontos de sensibilidade química específica presentes nos miracídios (Wilson e Denison, 1970). Sokolina (1983) diz que a composição bioquímica do muco estimula ou desestimula a atração e que na presença de fisídeos os miracídios rapidamente perdem a mobilidade e morrem. Preocupados com esse fenômeno, Nansen et al. (1976) sugeriram o uso de substâncias radioativas para tentar elucidar qual a influência física ou química do caramujo para que o miracídio o infectasse. Os trabalhos de Grigoryan (1965) e Grigoryan e Badeyan (1977) com Physa acuta assinalaram a existência de substância tóxica secretada por esses moluscos, que mata os miracídios de fasciolas em 20 a 30 minutos. Embora esta substância seja produzida na ausência dos miracídios, a presença destes aumenta a produção, por isso os autores sugeriram investigações nesse campo com vistas ao controle biológico.

Preveraud-Sindou e Rondelaud (1990) observaram que a presença de outros limneídeos que não o predominante da área e outros moluscos (fisídeos, bulinídeos e planorbídeos) podem influenciar na seleção de penetração dos miracídios, fazendo com que em $L$. truncatula a infecção seja levada a termo, com uma produção elevada de esporocistos. Abrous et al. (1998) verificaram que o baixo nível de infecções por $F$. hepatica em certas regiôes da França é devida à ausência de $L$. truncatula, que é o hospedeiro normal, mesmo que outros moluscos possam funcionar como hospedeiro intermediário. A idéia de controle biológico de trematódeos com moluscos que não se infectam com determinados miracídios foi também sugerida por Malek (1977) e Chernin (1968); Chernin e Perlstein (1969), trabalhando com Schistosoma mansoni Sambon, 1907, sugeriram que moluscos que não são hospedeiros específicos poderiam servir de iscas, tendo papel importante na epidemiologia e controle biológico.

Assim, algumas espécies, de acordo com o grau de infectividade, são consideradas de menor importância como hospedeiro intermediário de F. hepatica (Ueno e Watanabe, 1980; Boray, 1966; Rao, 1966; Sazanov, 1972; Rondelaud, 1980; Cruz-Reyes, 1982 e Bouix-Bousson et al. 1983) e que existe um grau de especificidade para o hospedeiro e mútua adaptação (Kendall 1964) como mostra uma resistência parcial de $L$. fuscus à infecção por $F$. hepatica, dificultando o desenvolvimento larvar e que apenas os jovens sustentaram o desenvolvimento do parasito Dreyfuss et al. (2000). Na região por nós trabalhada, F. hepatica é mais específica ao limneídeo do que ao hospedeiro vertebrado, $L$. columella é o hospedeiro com maior potencial de infectividade e que os fisídeos não funcionam como tal.

A determinação do qüinquagésimo sexto dia para avaliação, de um modo geral, não se afastou dos resultados de outros autores como Furmaga e Gundlach (1967), que encontraram o período de 42 a 63 dias, Roundelaud (1980) 42 dias, Ueta (1980) que observou que o número de moluscos mortos aumentava a partir do qüinquagésimo dia pós-infecção, BouixBousson et al. (1983) que consideraram como máximo o quadragésimo quinto dia.

Thomas (1883) disse que poucos moluscos sobrevivem à infecção artificial e que no laboratório, a infecção é mais fatal para o molusco do que para o ovino. Esta sobrevivência não está presa somente à adaptação parasito/hospedeiro, considerando-se que outras condições ideais estejam mantidas, 
mas a uma estreita relação com o número de miracídios/ molusco. Esse fato foi comprovado quando se obteve maior índice de mortalidade com infecções de cinco miracídios do que as com três, cuja diferença foi altamente significativa. A percentagem de infecção também sofreu a mesma influência e este fato identificou-se ao observado por Rondelaud e Barthe (1982) que, com infecções com um, dois, cinco, dez e vinte miracídios obteve, respectivamente, as seguintes percentagens: $60,56,32,30$ e 18. Bouix-Bousson et al. (1983) afirmaram que o número de sobreviventes diminuiu à medida que o número de miracídios/molusco aumentou. Também corroborando com essa observação, Nuernberg et al. (1983) disseram que, com mais de cinco miracídios, sobrevinha a morte do molusco antes da emergência de cercárias e Dreyfuss et al. (1999) disseram que, com até 20 miracídios, os moluscos sobrevivem à infecção, mas a maior produção de metacercárias ocorre com a infecção por um miracídio, sendo esta a mais efetiva e com média de sobrevivência do molusco maior.

A comprovação de que não houve influência do hospedeiro vertebrado do qual provinham os ovos corrobora a afirmação de Boray (1967) de que o hospedeiro vertebrado pouco representa na especificidade parasito/hospedeiro.

O fato de só terem sido conseguidas metacercárias pela dissecação dos moluscos para liberação das cercárias encontra correspondência na literatura especializada. Kendall \& McCullough (1951) observaram que a emergência de cercárias é passiva e resultante da atividade do manto, que causa o aumento da pressão na parte terminal do espaço visceral. Esses autores também referiram que, no campo, as chuvas e o movimento da água aumentavam a atividade do molusco favorecendo a emergência das cercárias. Boray (1963) confirmou esses autores, destacando que a emissão natural é melhor do que a experimental. Berghen (1964) também não obteve emissão de cercárias em condições laboratoriais e Hodasi (1972) consolidou afirmando que, nessas condições normais de laboratório, $L$. truncatula não fazia emergência total de cercárias. Ueta (1979), trabalhando com L. columella, só obteve metacercárias através do sacrifício dos moluscos com sinais de padecimento.

O aumento do crescimento das L. columella infectadas já observado por Berghen (1964) parece ser induzido pela presença das formas larvais em desenvolvimento, sendo apoiado na proposição de Wright (1971) e, segundo esse autor, o crescimento dos moluscos é conseqüente dos aumentos de volume interno da concha e essas mudanças resultam no aumento das proporções externas que poderiam ser usadas como índice superficial da maturidade reprodutiva. Considerando a pressão interna ocasionada pelo aumento do volume corporal do molusco parasitado, o mecanismo de crescimento da concha poderia ser similar ao descrito por Wright (1971). Outros autores também já assinalaram o fenômeno de gigantismo em suas observações como Rondelaud e Barthe (1978), que disseram que a distribuição de rédias de primeira geração poderia influenciar no crescimento e Wilson e Denison (1980), quando referiram maior tamanho para L. truncatula infectada. Jimenez-Albarran (1979), quando infectou $L$. truncatula com tamanhos de conchas variando entre 5,27 a $5,66 \mathrm{~mm}$, comparado com os testemunhos com variação de 5,23 a $5,62 \mathrm{~mm}$, obteve os aumentos 12,62 a 12,96 $\mathrm{mm}$ para os infectados e 10,41 a $10,81 \mathrm{~mm}$ para os controles. Bouix-Busson et al. (1985) referiram um crescimento rápido para moluscos infectados, salientando que o tamanho inicial não influencia no tamanho de pós-infecção.

$O$ fato de ter sido constatado que as $L$. columella infectadas com três miracídios tanto foram maiores que as nãoinfectadas, como das que foram infectadas com cinco miracídios, talvez seja reflexo de que, nessas infecções, à medida que era aumentado o número de miracídios/molusco, a sobrevivência diminuía, o que mantém identidade com o achado de Kawanaka (1978) e coerência com Combes (1982), que afirmou que a patogenicidade depende do número de miracídios que penetram no molusco.

\section{Agradecimento}

À Heloisa Diniz, do Laboratório de Produção e Processamento de Imagens / IOC / Fiocruz, pela assistência técnica com relação às figuras.

\section{Referências}

ABROUS, M., DREYFUSS, G., CABARET, J. Unusual transmission of the liver fluke, Fasciola hepatica, by Lymnaea glabra or Planorbis leucostoma in France. J. Parasitol., v. 84, n. 6, p. 1257-1259, 1998.

AMATO, S. B., REZENDE, H. E. B., GOMES, D. C., SERRA-FREIRE, N. M. Epidemiology of Fasciolosis hepatica in the Paraíba River Valley, São Paulo, Brasil. $11^{\text {th }}$ CONFERENCE OF WORLD ASSOCIATIONOF THE ADVANCEMENT OF VETERINARY PARASITOLOGY, 1985, Rio de Janeiro. Proceedings... 42, 1985.

AMATO, S. B., REZENDE, H. E. B., GOMES, D. C., SERRA-FREIRE, N. M. Epidemiology of Fasciola hepatica infection in the Paraiba River Valley, São Paulo, Brasil. Vet. Parasit., v. 11, p. 275-284, 1986.

BARTHE, D., RONDELAUD, D. Premières études sur la susceptibilité de trois especes de Physidae et de Bulinus truncatus Audouin a l'infestation fasciolienne. A propos de quelques observations histhologiques. Bull. Soc. Fr. Parasitol., v. 4, n. 1, p. 33-38, 1986.
BERGHEN, P. Some Lymnaeidae as intermediate host of Fasciola hepatica in Belgium. Exp. Parasitol., v. 15, p. 118-124, 1964.

BORAY, J. C. The ecology of Fasciola hepatica with particular reference to its intermediate host in Australia. $17^{\text {th }}$ WORLD VETERINARY CONGRESS, 1963, Hanover. Proceedings I: p. 709-715, 1963.

BORAY, J. C. Studies on the relative susceptibility of some lymnaeids to infection with Fasciola hepatica and on the adaption of Fasciola spp. Ann. Trop. Med. Parasitol., v. 60, n. 1, p. 114-124, 1966.

BORAY, J. C. Host-parasite relationship between Lymnaeid snails and Fasciola hepatica. Vet. Med. Rev., p. 132-140, 1967.

BOUIX-BUSSON, D., RONDELAUD, D., COMBES, C. Fasciola hepatica $L$.: Étude de facteurs influençant la durée de la période prépatente chez Lymnaea glabra Müller. Ann. Parasitol. Hum. Comp. v. 60, p. 5$10,1985$. 
BOUIX-BUSSON, D., RONDELAUD, D., PREVOST, J. Influence du nombre miracidiums et de l'age du mollusque sur la survie et le degré d'infestation de Lymnaea glabra Müller par Fasciola hepatica L. Ann. Parasitol. Hum. Comp., v. 58, n. 4, p. 347-352, 1983.

CHERNIN, E. Interference with the capacity of Schistosoma mansoni miracidia to infect the molluscan host. J. Parasitol., v. 54, n. 3, p. 509$516,1968$.

CHERNIN, E., PERLSTEIN, J. M. Further studies on interference with the host-finding capacity of Schistosoma mansoni miracidia. J. Parasitol., v. 55, n. 3 , p. 500-508, 1969.

COMBES, C. Trematodes: antagonism between species and sterilizing effects on snails in biological control. Parasitology, v. 84, p. 151-175, 1982.

CRUZ-REYES, A. Differential suitability of six species of lymnaeid snails exposed to Fasciola hepatica Linnaeus, 1758. 1982. 179 f. Ph. D. Disertation - Tulane University.

DREYFUSS, G., VIGNOLES, P., RONDELAUD, D., VAREILE-MOREL, C. Fasciola hepatica: Characteristics of infection in Lymnaea truncatula in relation to the number of miracidia at exposure, Exp. Parasitol., v. 92, n. 1, p. 19-23, 1999.

DREYFUSS, G., ABROUS, M., RONDELAUD, D. The susceptibility of Lymnaea fuscus to experimental infection with Fasciola hepatica. $J$. Parasitol., v. 86, n. 1, p. 158-160, 2000.

FURMAGA, S., GUNDLACH, J. L. Lymnaea stagnalis as a source of infection with Fasciola hepatica. Acta Parasitol. Polon., v. 15, n. 32 , p. 237-240, 1967.

GRIGORYAN, G. A. [Effect of fresh-water mollusc, Physa acuta, on miracidia of Fasciola.] Veterinaiya, v. 42, n. 12, p. 44-46, 1965. (Em russo).

GRIGORYAN, G. A., BADEYAN, G. [Some prerequisites for the biological control of Fasciola infections.] THIRD NATIONAL CONFERENCE OF PARASITOLOGY, 1977, Abena, Bulgaria. Oct-Abstract. p. 15-17, 1977. (Em russo).

HODASI, J. K. M. The output of cercariae of Fasciola hepatica by Lymnaea truncatula and the distribuition of metacercariae on grass. Parasitology, v. 64, n. 1, p. 53-60, 1972.

JIMENEZ-ALBARRAN, M. Estudios experimentales sobre biología de Fasciola hepatica: $3^{\circ}$ - Influencia del parasitismo sobre el crecimiento de Lymnaea (Galba) truncatula infestada con las fases larvarias de F. hepatica. Rev. Iber. Parasitol., v. 39, p. 95-105, 1979.

KAWANAKA, M. On the resistance of $L y m n a e a$ snails to infection with some larval trematodes. 1. Comparison of tissue reactions of snails $L$. ollula and $L$. japonica, exposed to Trichobilharzia brevis or Fasciola sp. Jap. J. Parasitol., v. 27, n. 3, p. 215-224, 1978.

KENDALL, S. B. Some factors influencing the development and behaviour of trematodes in their molluscan hosts. In: TAYLOR, A. E. R. (ed.) Host-Parasite relationships in invertebrates hosts. Oxford: Blackwell Scientific Publications, 1964, p. 297-329.

KENDALL, S. B., McCULLOUGH, F.S. The emergence of the cercariae of Fasciola hepatica from snail Lymnaea truncatula. J. Helminthol. v. 25, n. 1/2, p. 77-92, 1951.

LEUCKART, R. Zur Entwick lungsgeschichte des Leberegels (Distomum hepaticum). Arch. Natur., v. 48, p. 80-119, 1882.

MALEK, E. A. Studies on the Parasitism. J. Invert. Pathol., v. 29, p. 1-6, 1977.

NANSEN, P., CHRISTENSEN, N. O., FRANDSEN, F. A technique for in vivo labelling of Fasciola hepatica miracidia with radioselennium. $Z$. Parasitenk., v. 49, p. 73-80, 1976.

NUERNBERG, S., REZENDE, H. E. B., SERRA-FREIRE, N. M., GOMES, P. A., ARAÚJO, J. L. B. Biologia e susceptibilidade de Lymnaea cubensis (Mollusca, Lymnaeidae) a infecções por Fasciola hepatica em condições experimentais. Pesq. Vet. Bras., v. 3, n. 1, p. 1-10, 1983.
PARAENSE, W. L. Lymnaea viatrix and Lymnaea columella in the neotropical region: a distributional outline. Mem. Inst. Oswaldo Cruz, v. 77 , n. 2, p. 181-188, 1982.

PREVERAUD-SINDOU, M., RONDELAUD, D. Fasciola hepatica L.: Donnees experimentales sur l'attraction des miracidiums par Lymnaea truncatula Müller et des mollusques de familles voisines. Bull. Soc. Fr. Parasitol., v. 8, n. 2, p. 277-282, 1990.

RAO, C. M. P. On the comparative susceptibility of Lymnaea natalensis (Kraus) and L. rufescens (Gray) to infection with Fasciola gigantica (West African strain) and the tissue responses in snails. J. Helminthol., v. 40, n. 1/2, p.131-140, 1966.

REZENDE, H. E. B., ARAÚJO, J. L. B., GOMES, P. A. C., NUERNBERG, S., PIMENTEL NETO, M., OLIVEIRA, G. P., MELLO, R. P. Notas sobre duas espécies de Lymnaea Lamark, 1799, hospedeiros de Fasciola hepatica L. no Estado do Rio de Janeiro (Mollusca, Gastropoda, Basommatophora, Lymnaeidae). Arq. Univ. Fed. Rur. Rio de Janeiro, v. 3, n. 1 , p. $21-23,1973$.

RONDELAUD, D. Données épidémiologiques sur la distomatose humaine à la Fasciola hepatica $L$. dans la région du Limousin, France. Les plantes consomées et les limnées vectrices. Ann. Parasitol. Hum. Comp., v. 55, n. 4, p. 393-405, 1980.

RONDELAUD, D., BARTHE, D. Étude histologique du développement de Fasciola hepatica chez $L$ ymnaea truncatula, $L$. glabra et $L$. palustris infestées des leur naissance. C. R. Soc. Biol., v. 172, n. 6, p. 11941200, 1978.

RONDELAUD, D., BARTHE, D. Relationship of the amoebocyteproduction organ with the generalized amoebocytic reaction in Lymnaea truncatula Müller infected by Fasciola hepatica L. J. Parasitol., v. 68, n. 5, p. 967-969, 1982.

SAZANOV, A. M. [A protective response of lymnaeid molluscs to the penetration of Fasciola hepatica miracidia.] Trudy Useosoyuznogo Institula Gel'mintologii. In: SKRJABIN, K. I., 1972, p. 163-170. (Em russo).

SHIFF, C. J. Molluscan defense mechanisms: Immunity or population biology? Parasitology Today, v. 10, n. 5, p. 188-190, 1994.

SPIEGEL, M. R. Estatistica. São Paulo: McGraw-Hill do Brasil, 1976.

SOKOLINA, F. M. [On the intermediate host of Fasciola hepatica (factors determining specificity).] Severo-Osetinskij Gosudarstvenny, p. 2631, 1983. (Em russo).

THOMAS, A. P. The life history of the liver-fluke (Fasciola hepatica). Quart. J. Mic. Sci., v. 23, p. 99-133, 1883.

UENO, H., WATANABE, S. Ecological studies on the common liver-fluke in Japan. I. Comparison of intermediate hosts between the japanese native live fluke and Fasciola hepatica from England. Bull. N. I. A. H. v: 38, p. 167-181, 1980.

UETA, M. T. Comportamento de Lymnaea Say, 1817 (Gastropoda, Pulmonata) experimentalmente infectada com miracídios de Fasciola hepatica (Lineu, 1758) (Trematoda). CONGRESSO DA SOCIEDADE BRASILEIRA DE PARASITOLOGIA, 4., Campinas, SP, 1979. Anais... 58.

UETA, M. T. Infecção experimental de Lymnaea columella por Fasciola hepatica. Rev. Saúde Publ. São Paulo, v. 14, n. 1, p. 43-57, 1980.

WILSON, R. A. An investigation into the mucus produced by Lymnaea truncatula the snail host of Fasciola hepatica. Comp. Biochem. Physiol., v. 24, p. 629-663, 1968.

WILSON, R. A., DENISON, J. Short-chain fatty acids as stimulants of turning activity by the miracidium of Fasciola hepatica. Comp. Biochem. Physiol. v. 32, p. 511-517, 1970.

WILSON, R. A., DENISON, J. The parasitic castration and gigantism of Lymnaea truncatula infected with the larval stages of Fasciola hepatica. Z. Parasitenk., v. 61, n. 2, p. 109-113, 1980.

WRIGHT, C. A. Flukes and snails. New York: Hafner Press, 1971, p. 1168. 